\title{
Nested Protein Lattices in a Giant Phage Capsid Suggest Partial Maturation and a Residual Scaffold
}

Bernard Heymann ${ }^{1}$, Bing Wang ${ }^{2}$, William Newcomb ${ }^{3}$, Dennis Winkler ${ }^{3}$, Weimin Wu ${ }^{4}$, Erin Reilly ${ }^{5}$, Julie Thomas $^{5}$ and Alasdair Steven ${ }^{3}$

${ }^{1}$ NIAMS/NIH, Bethesda, Maryland, United States, ${ }^{2}$ NYU Langone Health, New York, New York, United States, ${ }^{3} \mathrm{NIH}$, Bethesda, Maryland, United States, ${ }^{4} \mathrm{NIH}$, Frederick, Maryland, United States, ${ }^{5}$ Rochester Institute of Technology, Rochester, New York, United States

"Giant" phages - also known as "jumbo" phages - have genomes in excess of >200 kbp dsDNA and correspondingly complex assembly pathways (Hendrix, 2009). One such, the Salmonella phage SPN3US, packages a $240 \mathrm{kbp}$ dsDNA genome into a capsid $\sim 1500 \AA$ in diameter (vertex-to-vertex), followed by attachment of a contractile tail. The head is composed of $\sim 50$ proteins, but only three are highly abundant: the major capsid protein, gp75, and the two ejection proteins, gp53 and gp54 (Ali, et al., 2017). Collective phage lore suggests that assembly should commence with formation of a procapsid guided by interactions between the growing surface shell and morphogenic scaffold. This is followed by activation of the protease, leading to expulsion of scaffold and major structural transformation (expansion) of the surface shell. We have used a protease-deficient mutant to probe earlier steps of the SPN3US head assembly pathway by cryo-EM.

Cells infected with an amber mutant in the prohead protease were harvested and lysed (Ali, et al., 2017) and capsids extracted by differential centrifugation followed by sucrose density centrifugation. SDSPAGE of this material showed three major bands which mass spectrometric analyses confirmed represented the precursor or uncleaved forms of the major capsid protein and the two ejection proteins (Figure 1B). We vitrified the specimens by standard techniques and imaged them in a Krios electron microscope (Thermo Fisher) at $300 \mathrm{kV}$ equipped with a K2 Summit direct detector and energy filter (Gatan). We performed reconstructions with Bsoft (Heymann, 2018).

The majority of these capsids projected a distinctive "mottled" pattern, quite different from that of the mature DNA-filled head (cf. Figure 1A). Reconstruction of the mottled capsid revealed that it is organized into three layers (Figure 1C). The outer shell has $\mathrm{T}=27$ icosahedral geometry (Figure 2A) as in some other giant phages, such as $\varphi \mathrm{KZ}(\mathrm{Wu}$, et al., 2012) and PBS1 (Hua, et al., 2017). Underlying this shell is a second thicker inner shell that makes contact with a subset of symmetry-related sites on the outer shell that correspond to a $\mathrm{T}=1$ lattice. However, this lattice has an unusual - to our knowledge, unprecedented - organization. In particular, it is markedly different from the "inner body" of phage $\varphi \mathrm{KZ}$ thought to be composed of ejection proteins ( $\mathrm{Wu}$, et al., 2012). Additional density is present inside the inner shell but is smeared into a continuum by averaging. Local resolution analysis (Figure 2) confirms that the structure is well defined for the outer capsid shell and inner shell but is much less so in the interior. Previous work on phage assembly suggests two possibilities for the role of the inner shell, a structure that is not present in the mature head. It may represent the morphogenic scaffold which would be expected to exit from a maturing procapsid but in this case - in the absence of proteolysis - may have been retained. Alternatively, it may represent an assembly of ejection proteins, retained in a disordered state in the DNA-packaged capsid, and destined for delivery into a host cell in the next cycle of infection. These options are not mutually exclusive. 
Acknowledgments. This research is supported by the National Institute of General Medical Sciences Award Number UA5GM126533, and by the NIAMS intramural research program, using the NIH Multi-Institute Cryo-EM Facility (MICEF).
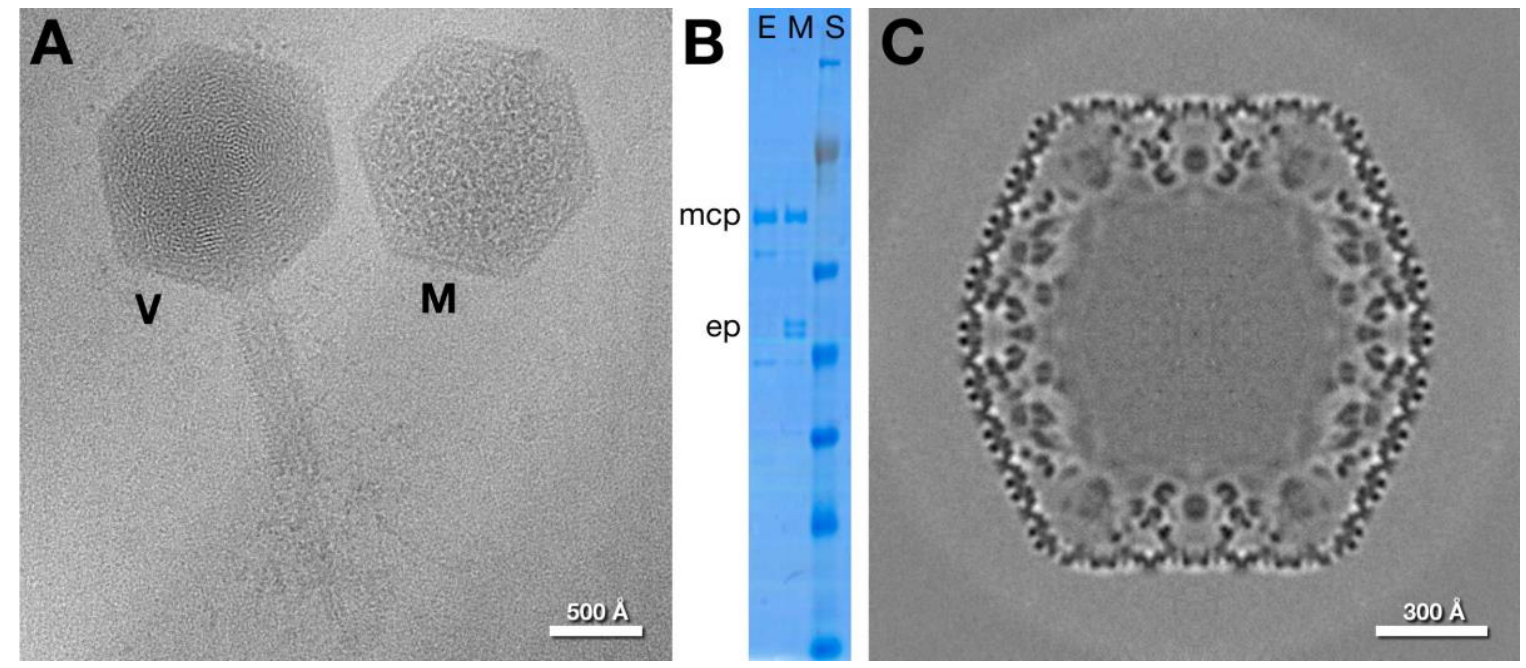

Figure 1. (A) A cryo-micrograph of a SPN3US virion with a DNA-packed head and tail (V) and a mottled capsid (M). (B) SDS-PAGE gel of empty (E) and mottled (M) capsids, showing bands for the major capsid protein (mcp) and the ejection proteins (ep). (C) Reconstruction of the $\sim 1500 \AA$ mottled capsid from 3000 particle images with a resolution of $\sim 14$ A. The mottled capsid has a thin outer shell and a complicated inner shell composed of an open network of densities.
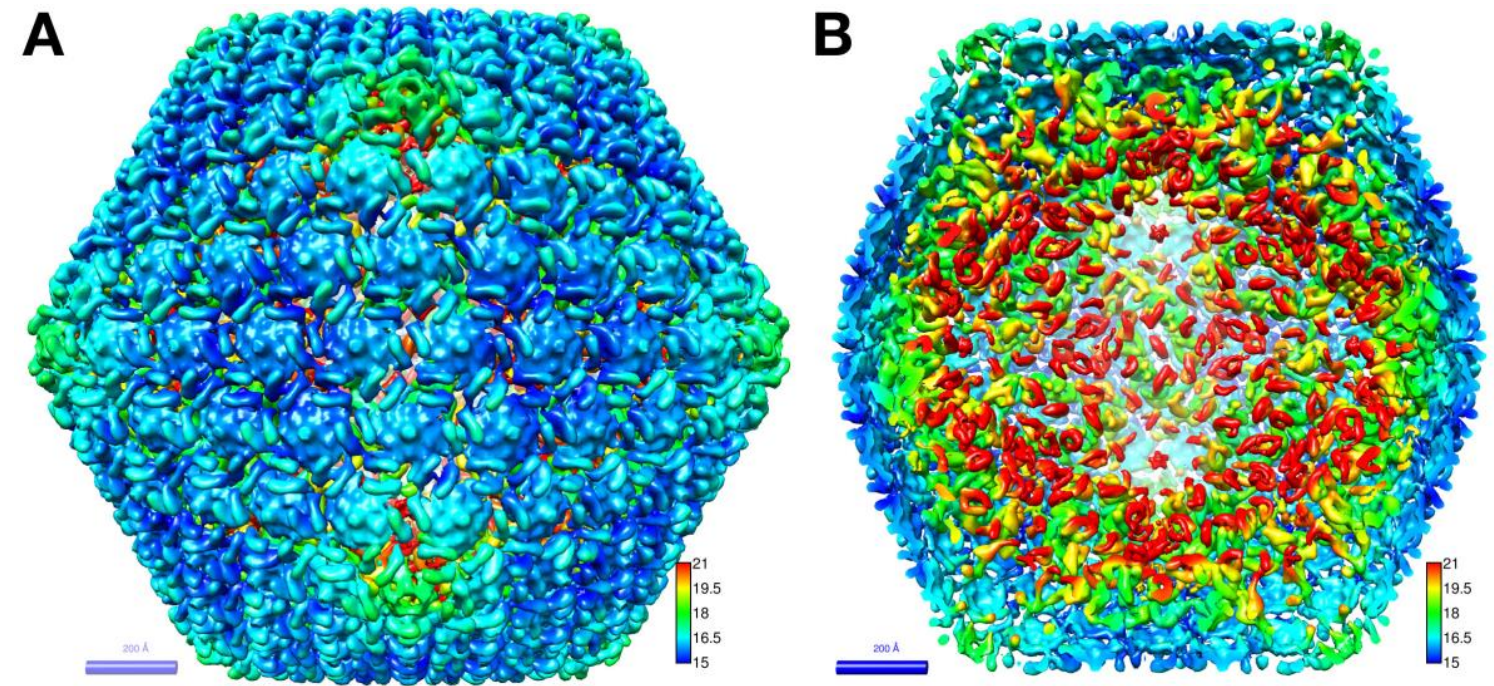

Figure 2. Isosurfaces of the mottled capsid reconstruction colored by local resolution estimates. (A) Outside view, showing the $\mathrm{T}=27$ icosahedral outer shell. (B) Cut through to show the $\mathrm{T}=1$ icosahedral inner shell, decreasing in local resolution towards the middle. Many hook-like densities are evident, likely many copies of the ejection proteins.

\section{References}

Ali, B., Desmond, M.I., Mallory, S.A., Benitez, A.D., Buckley, L.J., Weintraub, S.T., Osier, M.V., Black, L.W. \& Thomas, J.A. (2017). To Be or Not To Be T4: Evidence of a Complex Evolutionary Pathway of Head Structure and Assembly in Giant Salmonella Virus SPN3US. Front Microbiol 8, 2251. 
Hendrix, R.W. (2009). Jumbo bacteriophages. Curr Top Microbiol Immunol 328, 229-240.

Heymann, J.B. (2018). Guidelines for using Bsoft for high resolution reconstruction and validation of biomolecular structures from electron micrographs. Protein Sci 27(1), 159-171.

Hua, J., Huet, A., Lopez, C.A., Toropova, K., Pope, W.H., Duda, R.L., Hendrix, R.W. \& Conway, J.F. (2017). Capsids and Genomes of Jumbo-Sized Bacteriophages Reveal the Evolutionary Reach of the HK97 Fold. mBio 8(5).

Wu, W., Thomas, J.A., Cheng, N., Black, L.W. \& Steven, A.C. (2012). Bubblegrams reveal the inner body of bacteriophage phiKZ. Science 335(6065), 182. 